\title{
Income gradients within child and adolescent antisocial behaviours
}

Patrycja J. Piotrowska, Christopher B. Stride, Barbara Maughan, Robert Goodman,

Liz McCaw and Richard Rowe

\section{Background}

Low income is a widely studied risk factor for child and adolescent behavioural difficulties. Previous research on this relationship has produced mixed findings.

\section{Aims}

To investigate the level, shape and homogeneity of income gradients in different types of antisocial behaviour.

\section{Method}

A representative sample of 7977 British children and adolescents, aged 5-16 years, was analysed. Hypotheses concerning the shapes and homogeneity of the relationships between family socioeconomic status and multiple antisocial behaviour outcomes, including clinical diagnoses of oppositional-defiant disorder, conduct disorder and symptom subscales, such as irritability and hurtfulness, were tested by structural equation models.

\section{Results}

Consistent income gradients were demonstrated across all antisocial behaviours studied. Disorder prevalence and mean symptom counts decreased across income quintiles in a non-linear fashion.

\section{Conclusions}

Our findings emphasise that income gradients are similar across different forms of antisocial behaviour and indicate that income may lead to greater behavioural differences in the mid-income range and less variation at low- and high-income extremes.

\section{Declaration of interest}

R.G. is owner of Youthinmind Ltd which provides no-cost and low-cost software and websites related to the Development and Well-Being Assessment.

\section{Copyright and usage}

(c) The Royal College of Psychiatrists 2015.
Social inequalities have been extensively studied in relation to physical and mental health. Typically, a 'social gradient' has been identified, in which those of a higher socioeconomic status (SES) have better health outcomes than those with lower SES. This difference exists across the entire socioeconomic classification; for example, people just below the top do worse than those on the top. Social gradients have been shown in many physical disorders including coronary heart disease ${ }^{1-4}$ and high blood pressure. ${ }^{5} \mathrm{~A}$ systematic review of social inequalities in anxiety and depression reported that the majority of studies found an association between lower social status and higher prevalence of these disorders. ${ }^{6}$

A large number of studies have addressed the role of SES in antisocial behaviour. ${ }^{7}$ Many studies have found that children from low-SES backgrounds show higher prevalence rates or mean symptom counts of antisocial behavioural problems, ${ }^{8-11}$ although this relationship has not always been found. ${ }^{12}$ Most of these studies have treated SES as a dichotomy and examined the differences between the low- and high-SES groups; less attention has been paid to differences in prevalence across the entire range of economic advantage. Social gradients, however, have been documented by Dodge et al. ${ }^{13}$ They did find decreasing levels of externalising problems among pre-schoolers and young children with increases in socioeconomic class, suggesting that the difference exists not only between the poor and the better-off. A similar gradient in the prevalence of conduct disorder, with decreasing levels across income quintiles, was reported by Emerson et $a l^{14}$ among children and adolescents aged 5-15 years. However, such gradients have not been systematically studied across different forms of antisocial behaviour. This points towards the need for a thorough examination of gradients in behavioural disorders and its potential implications.

Antisocial behaviour is a heterogeneous concept and includes a wide range of disruptive and/or aggressive behaviours, from persistent rule-breaking through to physical aggression. To address in part the heterogeneity within antisocial behaviour, these behaviours can be grouped to form diagnostic criteria for two subtypes of antisocial behaviour: conduct disorder, which involves behaviours such as physical fighting, vandalism, stealing and lying; ${ }^{15}$ and oppositional-defiant disorder ${ }^{15}$ consisting of disobedience, irritability and hurtfulness. Furthermore, a distinction between physical aggression and non-physically aggressive rulebreaking may be made to subcategorise conduct disorder. ${ }^{16}$ Symptoms characterising the presence of oppositional-defiant disorder may be further subdivided to form symptom subscales, such as irritability, hurtfulness and headstrongness. ${ }^{17}$ More recently, research has indicated that the presence of callousunemotional traits, associated with low levels of empathy and guilt, ${ }^{18}$ might index a distinctive dimension of antisocial behaviour. ${ }^{19}$ Importantly, these aforementioned subtypes of antisocial behaviour have been shown to display heterogeneity in terms of their risk factors, prognosis and intervention response. ${ }^{20}$

In this paper, we aim to address the limitations in the current literature, (a) by examining the income gradients across heterogeneous antisocial outcomes and (b) by investigating the shapes of the effect of income on these behaviours. In a large-scale community sample of children and adolescents aged 5-16, we study whether social gradients differ between oppositional-defiant disorder and conduct disorder, and between their associated subscales and callous-unemotional traits.

\section{Method}

\section{Sample and data collection}

The data for this study were taken from the Mental Health of Children and Young People in Great Britain - 2004 survey (B-CAMHS04, 
2004); full study details are described elsewhere. ${ }^{21}$ In summary, a sample of 10486 eligible addresses, drawn from the Child Benefit Records (a centralised register of families receiving the state benefit for each child in the family, which at the time was provided universally) were approached for interview. Of these, 7977 (response rate $=76 \%$ ) responded with sufficient information for diagnostic classification. The remaining families either declined or could not be traced. Parents and young people aged 11 and older were interviewed alone. For the younger children, their parent report was collected. A teacher questionnaire was also sent out where parents provided consent; teacher data were collected for $78 \%$ of participants (6236 of 7977). All study procedures received multicentre ethics approval.

\section{Measures}

The interview schedule administered to parents and older children contained both demographic questions and a series of psychopathological assessments, which will be described in turn.

\section{Oppositional-defiant disorder and conduct disorder diagnoses}

The Development and Well-Being Assessment (DAWBA) 22 was administered to parents (and to children aged 11 and older). This includes forced choice questions complemented by open-ended questions to provide a bigger picture of behavioural symptoms or difficulties. A shorter version of the DAWBA was administered to teachers. The clinical utility of the DAWBA, and its ability to permit diagnoses without detailed assessment and discriminate community and clinical samples, has been demonstrated elsewhere. ${ }^{2,23}$ For each child, responses from all reporters were combined to generate diagnoses of presence or absence of oppositional-defiant disorder and conduct disorder, according to the criteria described in DSM-IV-TR. ${ }^{15}$ The use of cross-reporter data allowed behaviours that may not be exhibited at home to be captured. These diagnoses were then reviewed by experienced clinical raters who assessed the information from all sources and additional notes. In contrast to the DSM-IV-TR guidelines, ${ }^{15}$ both oppositional-defiant disorder and conduct disorder diagnoses were allowed for the same individual in the data-set. Given our desire to use both measures, and to address the high comorbidity shared by these two disorders, we recoded these diagnoses in line with the manual guidelines, so that where both oppositionaldefiant disorder and conduct disorder criteria were met, only a diagnosis of conduct disorder was given to the individual.

\section{Antisocial behaviour subscales}

Although oppositional-defiant disorder and conduct disorder diagnoses constitute the overall indication of a child's problematic behaviour, items within the Awkward and Troublesome Behaviour section of the DAWBA offered the opportunity to examine subtypes of antisocial behaviour in greater detail. These behavioural difficulty symptoms were assessed on a 3-point Likert scale: not true (0), partly true (1) and certainly true (2). When administering the DAWBA to parents and young people, 'skip rules', based on the relevant Strength and Difficulties Questionnaire $(\mathrm{SDQ})^{24}$ score and a screening question asked at the beginning of each symptom section, were used to omit questions if a diagnosis in a particular area was unlikely. Skip rules, however, were not in force for teachers; hence they provided more complete symptom-level data. Therefore, our symptom count data are based only on report of teachers.

Following DSM $-5^{25}$ and previous research on oppositionality, ${ }^{17}$ oppositional-defiant disorder symptom questions were grouped to form three subscales measuring irritability (e.g. temper tantrums, being angry and resentful), headstrongness (e.g. disobedience, arguing with adults) and hurtfulness (e.g. being spiteful). As suggested in the literature, ${ }^{16}$ conduct disorder symptom items were grouped to form aggressive and non-aggressive behaviour dimensions.

A parent-report measure of callous-unemotional traits was also administered alongside the DAWBA. This consisted of seven statements as described in Moran et al, ${ }^{26}$ each assessed by the parent on a 3-point Likert scale coded as not true (0), partly true (1) and certainly true (2). The questions included perceiving a child as cold-blooded or callous, shallow, not keeping promises and not being genuinely sorry if she or he hurt someone; for example, 'Has shallow and fast-changing emotions?', 'Genuine in expression of his/her emotions?'. This measure has been shown to be highly correlated at 0.81 with the callous-unemotional component of the Antisocial Process Screening Device. ${ }^{19}$

To confirm hypothesised grouping of these antisocial symptoms subscales into the six distinct dimensions described above, a series of confirmatory factor analyses (CFA) was performed with symptom questions treated as categorical indicators by weighted least square parameter estimates (WLSMV). The six-factor model $\left(\chi^{2}=1104.23\right.$, d.f. $=284$, comparative fit index $(\mathrm{CFI})=0.992$, root mean square error of approximation $($ RMSEA $)=0.023)$ gave a satisfactory fit under the fit index criteria suggested by $\mathrm{Hu}$ \& Bentler, ${ }^{27}$ and better fit than alternative potentially competing factor solutions such as: a three-factor model with relevant oppositional-defiant disorder and conduct disorder subscales as well as callous-unemotional traits treated as three separate factors $\left(\chi^{2}=1539.335\right.$, d.f. $\left.=296, \mathrm{CFI}=0.988, \mathrm{RMSEA}=0.028\right)$; and $\mathrm{a}$ one-factor model comprising a single general antisocial behaviour dimension measured by all items $\left(\chi^{2}=5015.703\right.$, d.f. $=299$, $\mathrm{CFI}=0.954$, RMSEA $=0.053$. Comparisons of the model fit indices showed that the hypothesised six-factor model fits the data best. The antisocial behaviour symptom items loading on to each factor presented good reliability in the current data-set $(\alpha \geqslant 0.70)$ with the exception of non-aggressive conduct symptoms $(\alpha=0.58)$ and callous-unemotional measure $(\alpha=0.67)$. (Note: for the purpose of computing descriptive statistics, observed scales scores were used.)

\section{Income}

Family income was used as a proxy measure of SES; caregivers were asked to indicate their annual household gross income from all sources on a 22-point ordinal scale; the values ranged from 'no source of income' (0) to ' $\mathfrak{E} 40000$ or more' (21). These were then grouped to form income quintiles as follows: $1 \mathrm{st}=$ no

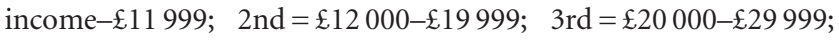

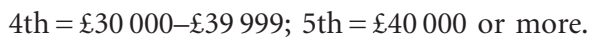

\section{Covariates}

B-CAMHS04 measured a number of demographic variables that might confound the relationship of SES and antisocial behaviour. As such, these were used as covariates in our analyses. Specifically, these were the number of children in a household; family type (coded 'married' (1), 'cohabiting' (2) or 'lone parent' (3)); the family's employment status ('both parents working' (1), 'one parent working' (2), 'neither parent working' (3)); caregiver's educational status ('No qualifications' (0), 'GCSE (D-F)' (1), 'GCSE (A-C)' (2), 'A-level' (3), 'Teaching/nursing qualification' (4), 'Degree level' (5)); child's age (in years); and gender ('female' (0), 'male' (1)).

\section{Data analyses}

Since oppositional-defiant disorder and conduct disorder diagnoses are systematically related to our symptom subscales (that is 
irritability, headstrongness, hurtfulness, aggressive and nonaggressive behaviours as well as callous-unemotional traits), the analyses were run as two separate series of models. In the first series, the clinical diagnoses of oppositional-defiant disorder and conduct disorder (both coded 'No diagnosis' (0), 'Diagnosis present' (1)) were the two outcomes modelled simultaneously, each predicted by income (coded into quintiles, which were modelled by four dummy variables) and adjusted for a range of household composition variables described above. This series of competing path analysis models were estimated by maximum likelihood with conventional standard errors. ${ }^{28}$ The second series of analyses used a set of structural equation models to test for finegrained differences between antisocial subtypes by using the six symptom subscales as outcomes, each symptom represented by a latent variable measured by the respective set of indicator items, with income quintiles and potentially confounding variables as the predictors (Fig. 1).

For both series of models, the testing comprised three stages. First, the shape of the income gradient (i.e. the effect of the income, coded into quintile groups) was formally tested by comparing the competing models described in Table 1. Specifically, we started with a free (unconstrained) model estimating free gradients for both outcomes, i.e. differences in oppositionaldefiant disorder (and conduct disorder) between any pair of quintiles were free to differ, and compared this against: a cubic model fixing the middle two quintile comparisons to be the same (i.e. a linear effect of income for those between bottom and top $20 \%$ of income); a curvilinear model, fixing the first three quintile comparisons to be the same and the last free; and a fully constrained model which forced a linear relationship between income and antisocial outcomes. We ascertained the best-fitting shape through comparing these nested models by either the Wald test of parameter constraints for oppositional-defiant disorder and conduct disorder; or the DIFFTEST procedure for antisocial subscales $^{28}$ (p. 625). Second, having established the shape of the income-antisocial behaviour relationship for both sets of outcomes, the invariance of the gradient was tested between the outcomes within each set (i.e. oppositional-defiant disorder $v$. conduct disorder; and between the six antisocial subscales, respectively). In this stage, we fitted competing models allowing entire gradients to differ across the outcomes (Model 1); fixing middle linear effect across the outcomes (Model 2); fixing the first quintile comparison ( $2 v .1)$ to be equal (Model 3a); fixing the last quintile comparison ( $5 v .4$ ) to be equal (Model 3b); and fixing all gradients to be equal across the outcomes (Model 4). Third, further exploratory analyses were conducted in which two other SES indicators; namely parental education and family employment status, were added to the best model for each set of outcomes emerging from stage 2. The effects of parental education and family employment were fixed to be the same across the outcomes and then tested against a less constrained model where their effects could differ between outcomes.

At each of the three stages in both series of models, income quintiles were dummy coded by backward difference contrasts so that each quintile other than the first was represented by a contrast providing a test of outcome levels between that quintile against the preceding quintile. Likewise, the effect of the income quintiles was always assessed when controlling for the potentially confounding demographic variables outlined above; unadjusted (i.e. without covariates) income results are presented in Tables 2 and 3. Because of incomplete data on key study measures, the sample sizes used in these sets of models were different; 6965 in the model predicting diagnoses of oppositional-defiant disorder and conduct disorder, and 5043 when the antisocial latent factors were the outcomes. Because of large sample size in the following analyses, the significance of the results was tested at three levels, namely $P<0.001, P<0.01$ and $P<0.05$. When directional hypotheses were tested, one-tailed significance values are reported.
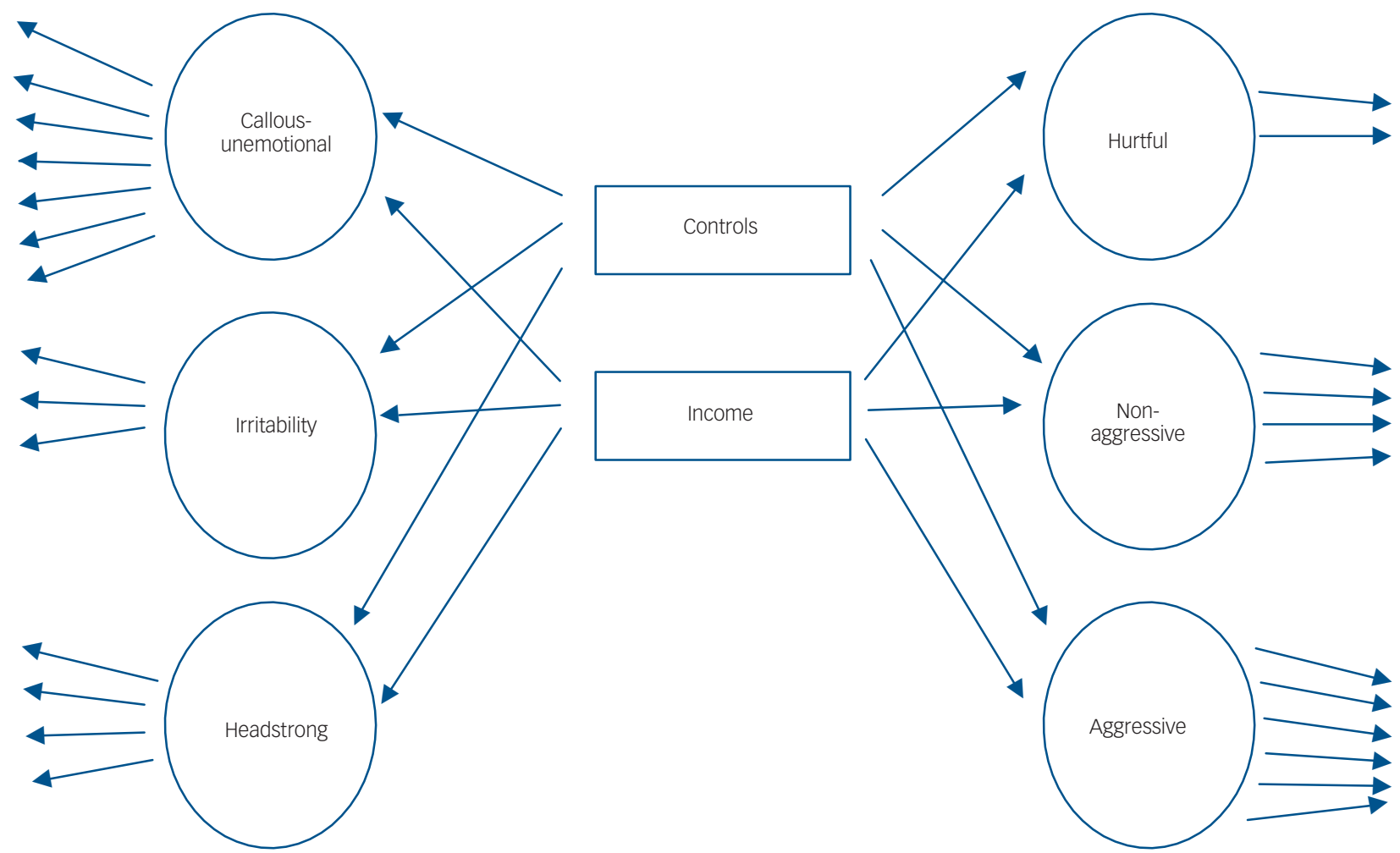

Fig. 1 Diagram of the structural equation model (SEM) for prediction of antisocial behaviour dimensions by income and potentially confounding variables. 
Where appropriate, the Akaike's information criterion (AIC; ${ }^{29}$ p. 402) is quoted as model fit indicator. Mplus $7.0^{28}$ was used in all analyses.

\section{Missing data}

Teacher report was used for symptom-level data measuring antisocial subscales; the characteristics of children excluded from the analyses because of missing information compared with the final sample are presented in Table DS1 (online supplement). Briefly, children with missing teacher data were significantly older, less likely to be brought up in wealthy neighbourhoods, have a carer with high degree and live with married parents. With the exception of age (Cohen's $d=0.296$ ), the effects sizes of these differences were small (Cramer's $V<0.06$ ). Response was not significantly related to gender and household size. However, children excluded from the analyses were more likely to have abnormal $(\geqslant 17 ; 24)$ SDQ Total Difficulties scores as reported by a parent (Cramer's $V=0.05$ ). These differences were not large and therefore are unlikely to affect the current study's findings. Consequently, listwise deletion was applied to all missing data.

\section{Results}

\section{Sample description}

A total of 7977 children (51.5\% boys) were initially included in this study, with mean age 10.54 years (range: 5-16). Oppositionaldefiant disorder and conduct disorder prevalence rates were $2.7 \%$ and $2.2 \%$ respectively. $\chi^{2}$-test indicated significant gender differences in prevalence rates; boys had higher rates of oppositional-defiant disorder $(3.5 v .1 .8 \%)$ and conduct disorder (2.8 v. 1.4\%) than girls (all $P<0.001)$. Similarly, significant gender differences were found for all other antisocial outcomes: callousunemotional traits, irritability, headstrongness, hurtfulness, aggressive and non-aggressive behaviours; with higher symptom mean counts among boys (Mann-Whitney $U, P<0.001)$. The mean scores for antisocial subscales as well as non-parametric (Spearman's Rank) correlations between the outcomes (ranging from 0.18 to 0.68 ) are presented in Table DS2 (online supplement).

\section{Income gradients and shape testing}

\section{oppositional-defiant disorder and conduct disorder}

The unadjusted prevalence rates of oppositional-defiant disorder and conduct disorder across income quintiles are presented in Table 4. The prevalence decreases with increasing income across the strata for both disorders showing a clear gradient, the only exception being between the 4 th and the 5 th income quintiles for oppositional-defiant disorder diagnosis.
As described in the data analysis section, the shape of the gradient was formally tested in a series of competing models including covariates; and the results are presented in Table 1. The Wald test of parameter constraints showed that the cubic shape fitted the data best, and alternative models of curvilinear or linear shapes were significantly worse than the free (i.e. baseline) model. This finding suggests that gradients are non-linear; there is a linear effect for the two middle comparisons (i.e. 3rd and 2nd quintile, and 4th and 3rd), suggesting parallel improvement in behavioural outcomes as family moves to a higher income quintile. This effect, however, does not hold at low- and highincome extremes where less variation is present. For ease of interpretation, the gradient results were plotted against the probability of each symptom occurring (as opposed to the odds) and are presented in Figs. DS1 and DS2 respectively (online supplement). These figures show differences in the probability of oppositional-defiant disorder or conduct disorder diagnoses across income quintiles, with the steepest changes in probability occurring in comparisons of the three middle-income quintiles.

\section{Antisocial subscales}

Table 4 presents social gradients in the antisocial behaviour subscales showing the gradual decrease in mean symptom count alongside increasing income. This trend was found across the entire socioeconomic classification and across all antisocial outcomes studied. In all inferential analyses, latent antisocial factors were used and separate models run to test four alternative shapes (Table 1). All models had satisfactory fit (CFI $>0.95$, RMSEA < 0.06); the cubic model was not significantly worse than the free baseline model. In contrast, both curvilinear and linear models were significantly worse, showing that a cubic-like shape fits income gradients in antisocial behaviour best. These significant differences held when curvilinear and linear models were compared against the cubic model $(P<0.01)$. These findings are of similar shape to the gradient described for diagnoses of oppositional-defiant disorder and conduct disorder.

\section{Are gradients heterogeneous across antisocial outcomes?}

\section{Oppositional-defiant disorder and conduct disorder}

To test whether income gradients differ between oppositionaldefiant disorder and conduct disorder, the free cubic model including previously mentioned covariates was first estimated for both outcomes, allowing gradients to differ across the outcomes. Income gradients were then constrained to be equal for both outcomes (i.e. the null hypothesis) and tested in a range of intermediate steps as described in the data analysis section.

Table 1 Competing model testing of the shape of the gradients in oppositional-defiant disorder and conduct disorder, and antisocial subscales

\begin{tabular}{|c|c|c|c|c|c|c|}
\hline & & \multicolumn{3}{|c|}{ Oppositional-defiant disorder and conduct disorder } & \multicolumn{2}{|c|}{ Antisocial subscales } \\
\hline & & $\chi^{2}$ (d.f.) & AIC & Wald test (d.f.) & $\chi^{2}$ (d.f.) & $\chi^{2}$ difference test (d.f.) \\
\hline Free & MW & 1398.02 (34) & 2864.04 & & 1740.81 (604) & \\
\hline Cubic & & $1398.24(32)$ & 2860.48 & $0.45(2)$ & $1749.72(610)$ & $3.79(6)$ \\
\hline Curvilinear & & $1404.56(30)$ & 2869.11 & $13.16(4)^{*}$ & $1769.62(616)$ & $24.73(12)^{\star}$ \\
\hline Linear & & $1406.69(28)$ & 2869.38 & $16.34(6)^{*}$ & 1777.53 (622) & $40.24(18)^{\star *}$ \\
\hline
\end{tabular}


Starting with the free model (Model 1), certain parts of the cubic gradients were subsequently fixed to be equal across both outcomes (Models 2, 3a and 3b); finally, the fully fixed model (Model 4) was estimated (Table DS3, online supplement). The Wald test of parameter constraints comparing the fully fixed (i.e. Model 4) to the free model (i.e. Model 1) produced a result of 3.56 (3), $P=0.31$, which indicates there were no significant gradient differences between these two disorders. Further constraint of parental education and family employment status to be the same across the outcomes was non-significant $(P>0.05)$. This suggests that the updated model is not significantly worse than the less constrained one; hence these additional constraints were included in the final model. Complete model results of the most parsimonious (i.e. fixed) model are presented in Table 2, and the free model with no constraints imposed is presented in Table DS4 (online supplement). After controlling for potentially confounding variables, significant differences were found between the first four quintiles in the model fixing oppositional-defiant disorder and conduct disorder gradients to be the same. It has been found that children from higher quintiles were significantly less likely to be diagnosed with oppositional-defiant disorder and conduct disorder than children in the preceding (i.e. lower) income quintile. This trend was maintained between the 4th and the 5th quintiles, yet was no longer significant after including covariates in the model. Odds ratios show that children in the 2 nd quintile are $26 \%$ less likely to be diagnosed than those in the first quintile; this further increased to $51 \%$ for the comparisons between the 3rd and the 2nd quintiles as well as the 4th and the 3rd. Finally, children from the most affluent families were $22 \%$ less likely to have the disorder than those in the 4 th quintile. Two other SES indicators were fixed across the outcomes showing that having both or one parent working ( $v$. none) is associated with a significantly lower probability of being diagnosed with oppositional-defiant disorder or conduct disorder. A similar trend was found for parental education, where higher qualifications were associated with better behavioural outcomes.

\section{Antisocial subscales}

The best-fitting cubic shape for six latent antisocial factors, as described in an earlier section was tested across six antisocial outcomes to examine whether income gradients differ across heterogeneous forms of antisocial behaviour. As described previously, the DIFFTEST was used to compare a tested model against the free model (i.e. Model 1). As shown in Table DS3 (online supplement), none of the tested models were significantly worse than the free model which allowed cubic gradients to differ between the outcomes. This suggests that cubic-like gradients do not significantly differ across antisocial subscales and the fixed and the most parsimonious model is satisfactory. Detailed model

Table 2 Constrained (fixed across outcomes) income logistic regression model predicting oppositional-defiant disorder and conduct disorder $(n=6965)^{\mathrm{a}}$

\begin{tabular}{|c|c|c|}
\hline & \multicolumn{2}{|c|}{ Odds Ratios $(95 \% \mathrm{Cl})^{\mathrm{b}}$} \\
\hline & Adjusted & Unadjusted \\
\hline Quintile 2 v. 1 & $0.74(0.58-0.93)$ & $0.44(0.37-0.52)$ \\
\hline Quintile 3 v. 2 & $0.49(0.35-0.68)$ & $0.25(0.19-0.32)$ \\
\hline Quintile 4 v. 3 & $0.49(0.35-0.68)$ & $0.25(0.19-0.32)$ \\
\hline Quintile 5 V. 4 & $0.78(0.60-1.01)$ & $0.53(0.42-0.67)$ \\
\hline \multicolumn{3}{|c|}{$\begin{array}{l}\text { Log likelihood }=-1407.21 \text {, Akaike's information criterion }=2858.42 \text {. } \\
\text { a. Estimates for the covariates included in the model available from the corresponding } \\
\text { author. } \\
\text { b. Bonferroni-corrected significant }(P<0.05) \text { results are presented in bold. }\end{array}$} \\
\hline
\end{tabular}

results are presented in Table 3 with all income as well as parental education and family employment status effects fixed across the outcomes. Additionally constraining the other SES indicators (i.e. parental education and family employment) to be equal across the outcomes did not significantly worsen the model fit; hence these estimates are fixed in the final model. All the quintile comparisons were significant and negative, suggesting lower antisocial scores for children from higher income families irrespective of the type of behaviour measured. This was also true before adjustment for relevant covariates, although coefficients were larger in magnitude. As in the first set of models, having at least one parent employed or higher qualifications were associated with lower levels of antisocial behaviour among children. The free (unconstrained) model results are presented in Table DS5 (online supplement).

\section{Discussion}

The primary aim of this study was to investigate the form of income gradients across different forms of antisocial behaviour. The B-CAMHS04 survey has a number of strengths for addressing this aim. These include a large sample size from the whole income range and derived from across Great Britain, with detailed measurement of a range of antisocial behaviour constructs.

\section{Non-linear income gradients}

As hypothesised, clear income gradients were found among all antisocial behaviours studied; differences in the prevalence of disruptive behaviours exist across the entire socioeconomic classification. There is no threshold below which all behavioural problems accumulate and above which there is an equally low level

Table 3 Unstandardised income regression coefficients (B) with $95 \%$ confidence intervals (CIS) of the cubic model fixed across latent factors $(n=5043)^{\mathrm{a}}$

\begin{tabular}{l}
\cline { 2 - 3 } \\
\cline { 2 - 3 }
\end{tabular}

Table 4 Unadjusted prevalence rates of oppositional-defiant disorder and conduct disorder and symptom mean counts for antisocial subscales by income quintiles

\begin{tabular}{|lccccc|} 
& \multicolumn{5}{c|}{ Quintiles } \\
\cline { 2 - 6 } & $\begin{array}{c}1 \text { 1st } \\
\text { (low) }\end{array}$ & 2nd & 3rd & 4th & $\begin{array}{c}\text { 5th } \\
\text { (high) }\end{array}$ \\
$\begin{array}{l}\text { Oppositional-defiant } \\
\text { disorder, \% }\end{array}$ & 4.60 & 3.60 & 2.30 & 1.00 & 1.40 \\
\hline Conduct disorder, \% & 4.20 & 3.30 & 1.50 & 0.90 & 0.70 \\
\hline Callous-unemotional traits & 2.13 & 1.76 & 1.48 & 1.25 & 1.09 \\
\hline Irritability & 0.85 & 0.72 & 0.49 & 0.41 & 0.39 \\
\hline Headstrong & 1.19 & 0.99 & 0.70 & 0.53 & 0.52 \\
\hline Hurtful & 0.40 & 0.35 & 0.23 & 0.17 & 0.17 \\
\hline Aggressive & 0.44 & 0.37 & 0.23 & 0.15 & 0.14 \\
\hline Non-aggressive & 0.33 & 0.25 & 0.15 & 0.09 & 0.10 \\
\hline
\end{tabular}


of such difficulties. After adjusting for a range of covariates, such as marital status or number of children in family, income remained a significant predictor of all antisocial behaviours studied and similar gradients were found. In some ways, the income gradient in callous-unemotional traits might be seen as unexpected because these traits have been found to be highly heritable. ${ }^{30}$ Children with callous-unemotional traits, however, constitute a heterogeneous group with low-anxious primary and high-anxious secondary variants of callous-unemotional traits (e.g. Skeem et al $^{31}$ ) which may have distinct aetiologies, primarily genetic and environmental respectively. The existence of clear income gradients and significant environmental predictors of callous- unemotional traits in this study (e.g. marital status, parental education) may suggest the predominance of secondary callous-unemotional traits in our community sample.

Our findings showed that the effect of income on behavioural problems is monotonic and there is a continuous decrease in disorder prevalence rates or mean symptom counts; the effect was found to be non-linear. As previously described, rates of such problems are generally low in community samples and behaviour simply cannot continuously improve with increases in income. On the other hand, despite decreases in income at the low extreme, levels of behaviour problems seem to flatten off (as showed in the best-fitting model for both sets of outcomes). This indicates that income differentially affects specific income groups tested in the model; below a certain low-income level, less money has a relatively smaller effect on antisocial behaviour outcome measures. These effects, however, are net of other potential confounds and mechanisms likely to be involved in explaining the association between income and antisocial behaviours; ${ }^{13}$ this warns further investigation.

Only a few past studies have explicitly investigated the idea of gradients in child and adolescent behavioural problems (e.g. Dodge et $a l^{13}$ Emerson et $\left.a l^{14}\right)$, with the former indicating a linear decrease in teacher-reported externalising problems across increasing SES as measured by the Hollingshead's four-factor index (i.e. parental education and occupation). In this study, we found a cubic-shaped model fit the data best for all antisocial dimensions tested. There was a linear decrease in antisocial behaviour for the middle-income categories, with less pronounced effect at both extremes. However, income quintiles cannot be directly compared with the Hollingshead SES index based on parental education and occupation which does not necessarily reflect the actual income. This could explain the discrepancy between the shape of previously identified gradients ${ }^{13}$ and those in the present work. Alternatively, this could also be accounted for by the substantially larger sample size in the current study providing the power to detect non-linear effects.

As outlined in the introduction, existing evidence on the association between income and broad operationalisations of antisocial behaviour presents mixed results with some studies reporting income as a significant predictor (e.g. Herrenkohl et $a l,{ }^{32}$ Tremblay et $a l^{33}$ ) and some suggesting otherwise (e.g. Holmes et $\left.a l^{34}\right)$. One possibility is that when looking at the effects of income on behavioural disorders, sampling from a wide range of incomes may be important. Given the cubic trends that we have identified, a weaker effect of income in studies focussing on low-SES or deprived samples would be expected (e.g. Keenan \& Shaw ${ }^{35}$ ).

\section{Gradients across antisocial behaviours}

When gradients were tested across the outcomes, no significant differences were found in either set of models, indicating the similarity of the gradients. Despite the heterogeneous nature of antisocial behaviour, income showed the same effect on all behaviours included in the current study when correlations between the outcomes were allowed in the model. This suggests that the similarity of the gradients does not result from the between-measure correlations, which were small to moderate in size in the analysis sample.

In the final set of analyses, an additional constraint of the two SES indicators (i.e. parental education and family employment status) was tested. The best-fitting model showed that the effects of these parental factors are not only significantly associated with antisocial behaviour but also seem to exhibit the same effect on different forms of antisocial behaviour. This was an exploratory analysis, however, and this finding requires further investigation into the relations between a range of SES indicators and antisocial behaviour as well as potential gradient tendencies.

\section{Strengths and weaknesses}

Our study provides a strong basis for addressing social gradients in a range of antisocial behaviours as well as potential differences between such gradients. However, the results must be considered in the light of a number of limitations. It was a cross-sectional study which does not enable us to definitively identify causal relationships. A further limitation concerns the categorical measurement of income; a continuous measure of income was not available in the B-CAMHS04 data-set. Some of our analyses, in particular those investigating the shape of the gradients would have benefited from a continuous income measure allowing estimation of polynomial shape parameters. In some ways, however, quintiles are less vulnerable to bias when studying income gradients as errors of estimation of income within quintiles (e.g. omission of benefits) would not affect the gradients; the order of families remains preserved. Also, teacher data representing antisocial subscales may not capture all the antisocial behaviours that children engaged in and it remains in question whether similar gradients can be found when using data from other informants in particular children and adolescents themselves. Finally, future studies should explore the nature of income gradients in antisocial behaviour subtypes that could not be differentiated in the data-set, such as those based on developmental trajectory. ${ }^{36}$

\section{Implications}

Our findings highlight the importance of studying samples with a full range of income to explore the effects that SES may have on all children, not only on those from the most deprived families. Furthermore, reducing inequalities, especially by boosting the wealth of those on low income, will likely improve behavioural outcomes. This also points towards the need to identify the factors that increase the risk for behavioural problems in families with low and medium incomes so that interventions can be targeted to these particularly vulnerable socioeconomic groups in order to reduce behavioural problems in the population. It remains unclear how certain SES groups respond to mental healthcare services and interventions and what programmes may be most effective. However, previous research suggests that individuals with low and medium incomes are less likely to access mental health services. ${ }^{37,38}$ Together with our findings highlighting significant differences in prevalence rates as well as the level of antisocial symptoms across income groups, it is important to increase the access to mental health services among these groups.

Patrycja J. Piotrowska, PhD, Christopher B. Stride, PhD, CStat, University of Sheffield, Western Bank, Sheffield, UK; Barbara Maughan, PhD, Robert Goodman FRCPsych, PhD, Institute of Psychiatry, Psychology \& Neuroscience, King's College London, UK; Liz McCaw, MSC, Richard Rowe, PhD, University of Sheffield, Western Bank, Sheffield, UK

Correspondence: Patrycja J. Piotrowska, University of Sheffield, Western Bank, Sheffield S10 2TP, UK. Email: pj.piotrowska@sheffield.ac.uk

First received 17 Dec 2013, final revision 19 Dec 2014, accepted 19 Dec 2014 


\section{References}

1 Marmot M, Rose G, Shipley M, Hamilton PJS. Employment grade and coronary heart-disease in British civil-servants. J Epidemiol Community Health 1978; 32: 244-9.

2 Marmot M, Brunner E. Cohort profile: the Whitehall II study. Int J Epidemiol 2005; 34: 251-6.

3 Marmot M, Shipley MJ, Rose G. Inequalities in death - specific explanations of a general pattern. Lancet 1984; 1: 1003-6.

4 van Rossum CTM, Shipley MJ, van de Mheen H, Grobbee DE, Marmot MG. Employment grade differences in cause specific mortality. A 25 year follow up of civil servants from the first Whitehall study. J Epidemiol Community Health 2000; 54: 178-84.

5 Colhoun HM, Hemingway H, Poulter NR. Socio-economic status and blood pressure: an overview analysis. J Hum Hypertens 1998; 12: 91-110.

6 Fryers T, Melzer D, Jenkins R. Social inequalities and the common mental disorders - a systematic review of the evidence. Soc Psychiatry Psychiatr Epidemiol 2003; 38: 229-37.

7 Piotrowska PJ, Stride CB, Rowe R. Social gradients in child and adolescent antisocial behavior: a systematic review protocol. Syst Rev 2012; 1: 38.

8 Hartas D. The ecology of young children's behaviour and social competence: child characteristics, socio-economic factors and parenting. Oxford Rev Edu 2011; 37: 763-83.

9 Amone-P'Olak K, Burger H, Huisman M, Oldehinkel AJ, Ormel J. Parental psychopathology and socioeconomic position predict adolescent offspring's mental health independently and do not interact: the TRAILS study. $J$ Epidemiol Community Health 2011; 65: 57-63.

10 Pagani L, Boulerice B, Vitaro F, Tremblay RE. Effects of poverty on academic failure and delinquency in boys: a change and process model approach. J Child Psychol Psychiatry 1999; 40: 1209-19.

11 Costello EJ, Compton SN, Keeler G, Angold A. Relationships between poverty and psychopathology - a natural experiment. JAMA 2003; 290: 2023-9.

12 Burt KB, Harold G, Hay DF, Pawlby S, Sharp D. The prediction of disruptive behaviour disorders in an urban community sample: the contribution of person-centred analyses. J Child Psychol Psychiatry 2004; 45: 1159-70.

13 Dodge KA, Pettit GS, Bates JE. Socialization mediators of the relation between socioeconomic-status and child conduct problems. Child Dev 1994; 65: 649-65.

14 Emerson $\mathrm{E}$, Graham $\mathrm{H}$, Hatton $\mathrm{C}$. Household income and health status in children and adolescents in Britain. Eur J Public Health 2006; 16: 354-60.

15 American Psychiatric Association. Diagnostic and Statistical Manual of Mental Disorders. Fourth Edition, Text Revision (DSM-IV-TR). APA, 2000

16 Burt SA. Are there meaningful etiological differences within antisocial behavior? Results of a meta-analysis. Clin Psychol Rev 2009; 29: 163-78.

17 Stringaris A, Goodman R. Three dimensions of oppositionality in youth. J Child Psychol Psychiatry 2009; 50: 216-23.

18 Frick PJ, White SF. Research review: the importance of callous-unemotiona traits for developmental models of aggressive and antisocial behavior. $J$ Child Psychol Psychiatry 2008; 49: 359-75.

19 Moran P, Rowe R, Flach C, Briskman J, Ford T, Maughan B, et al. Predictive value of callous-unemotional traits in a large community sample. J Am Acad Child Adolesc Psychiatry 2009; 48: 1079-84.
20 Burke JD, Loeber R, Birmaher B. Oppositional defiant disorder and conduct disorder: a review of the past 10 years, part II. J Am Acad Child Adolesc Psychiatry 2002; 41: 1275-93.

21 Green H, McGinnity A, Meltzer H, Ford T, Goodman R. Mental Health of Children and Young People in Great Britain, 2004. Palgrave Macmillan, 2005.

22 Goodman R, Ford T, Richards H, Gatward R, Meltzer H. The development and well-being assessment: description and initial validation of an integrated assessment of child and adolescent psychopathology. J Child Psychol Psychiatry 2000; 41: 645-55.

23 Foreman $D$, Morton $S$, Ford $T$. Exploring the clinical utility of the Development And Well-Being Assessment (DAWBA) in the detection of hyperkinetic disorders and associated diagnoses in clinical practice. J Child Psychol Psychiatry 2009; 50: 460-70.

24 Goodman R. The strengths and difficulties questionnaire: a research note. J Child Psychol Psychiatry 1997; 38: 581-6.

25 American Psychiatric Association. Diagnostic and Statistical Manual of Mental Disorders (DSM-5). APA, 2013.

26 Moran P, Ford T, Butler G, Goodman R. Callous and unemotional traits in children and adolescents living in Great Britain. Br J Psychiatry 2008; 192: 65-6.

27 Hu LT, Bentler PM. Fit indices in covariance structure modeling: sensitivity to underparameterized model misspecification. Psychol Methods 1998; 3: $424-53$.

28 Muthén LK, Muthén BO. Mplus User's Guide (7th edn). Muthén \& Muthén, 1998-2012.

29 Baguley T. Serious Stats: A Guide to Advanced Statistics for the Behavioral Sciences. Palgrave Macmillan, 2012

30 Viding E, Blair RJR, Moffitt TE, Plomin R. Evidence for substantial genetic risk for psychopathy in 7-year-olds. J Child Psychol Psychiatry 2005; 46: 592-7.

31 Skeem JL, Poythress N, Edens JF, Lilienfeld SO, Cale EM. Psychopathic personality or personalities? Exploring potential variants of psychopathy and their implications for risk assessment. Aggression Violent Behav 2003; 8 513-46.

32 Herrenkohl TI, Guo H, Kosterman R, Hawkins JD, Catalano RF, Smith BH. Early adolescent predictors of youth violence as mediators of childhood risks. J Early Adolescence 2001; 21: 447-69.

33 Tremblay RE, Nagin DS, Seguin JR, Zoccolillo M, Zelazo PD, Boivin M, et al. Physical aggression during early childhood: trajectories and predictors. Pediatrics 2004; 114: E43-50.

34 Holmes EK, Jones-Sanpei HA, Day RD. Adolescent outcome measures in the NLSY97 family process data set: variation by race and socioeconomic conditions. Marriage Family Rev 2009; 45: 374-91.

35 Keenan K, Shaw DS. The development of aggression in toddlers: a study of low-income families. J Abnorm Child Psychol 1994; 22: 53-77.

36 Moffitt TE. Adolescence-limited and life-course-persistent antisocial behavior: a developmental taxonomy. Psychol Rev 1993; 100: 674-701.

37 Wang PS, Lane M, Olfson M, Pincus HA, Wells KB, Kessler RC. Twelve-month use of mental health services in the United States: results from the national comorbidity survey replication. Arch Gen Psychiatry 2005; 62: 629-40.

38 Cohen P, Hesselbart CS. Demographic factors in the use of children's mental health services. Am J Public Health 1993; 83: 49-52. 